\title{
Vehicle model response in frequency domain
}

\author{
Jozef Melcer ${ }^{1}$ and Veronika Valašková, ${ }^{1, *}$ \\ ${ }^{1}$ University of Žilina, Faculty of Civil Engineering, Univerzitná 8215/1, 01026 Žilina, Slovak \\ Republic
}

\begin{abstract}
The offered article deals with one of the possibilities of numerical analysis of the vehicle response in frequency domain. It works with quarter model of vehicle. For the selected computational model of vehicle it quantifies the Frequency Response Functions (FRF) of both force and kinematic quantities. It considers the stochastic road profile. The Power Spectral Density (PSD) of the road profile is used as input value for the calculation of Power Spectral Density of the response. Al calculations are carried out numerically in the environment of program system MATLAB. When we know the modules of FRF or the Power Response Factors (PRF) of vehicle model the calculation of vehicle response in frequency domain is fast and efficient.
\end{abstract}

\section{Introduction}

A roadway is a typical structure subject to the dynamic effects of moving vehicles. The problem of rough surface road profiles and its influence on vehicle unwanted vibrations due to kinematic excitations is still a subject of research among automotive manufacturers and research groups, whose objective is to minimize their effects on the driver and passengers. Unevenness on the surface of the pavement is the main source of kinematic excitation of vehicle. The real load acting on the roads is variable in time and in frequency composition. This should be known for the solution of many engineering tasks as lifetime, design, reliability, structure development, micro tremor, etc [1-3]. This article presents simplest way how to obtain the Power Spectral Density (PSD) of input signal (in our case it is PSD of road unevenness), how to calculate the dynamic properties of analysed system (specifically the Power Response Factors) and PSD of output signals of individual quantities.

\section{Computational model of vehicle}

The discrete (lumped mass) computational models of vehicles can be created on three qualitative different levels: 1D - quarter model, 2D - plane model and 3D - spatial model. Every model has its advantages and disadvantages and under certain assumptions, it can be used for the solution of real practical problems. Quarter model of vehicle is shown in Fig. 1. It can be used to model the effects of half of one axle of the vehicle on a traffic road and under certain simplifying assumptions to model the effects of the entire vehicle. The

\footnotetext{
*Corresponding author: veronika.valaskova@fstav.uniza.sk
} 
disadvantage of this model is that it can only model the heave effect of the upper mass on the contact forces between the wheel and the runway. It cannot model the pitch effect not the roll effect of the suspended mass on the contact force between the wheel and the runway.

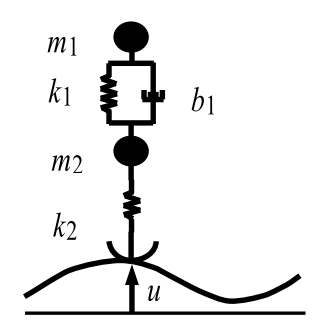

Fig. 1. Quarter model of vehicle.

The relation between the components of displacements $\{\mathbf{r}(t)\}$, corresponding to individual degrees of freedom, and deformations of joining elements $\{\mathbf{d}(t)\}$ gets the transpose static matrix $[\mathbf{A}]^{\mathbf{T}}$

$$
\{\mathbf{d}(t)\}=[\mathbf{A}]^{\mathbf{T}} \cdot\{\mathbf{r}(t)\}
$$

Dependence between elastic forces in joining elements (in the sense of action of mass objects on jointing members) and its deformations is described by the equation

$$
\left\{\mathbf{F}_{\mathbf{r e}}(t)\right\}=[\mathbf{k}] \cdot\{\mathbf{d}(t)\}
$$

where $[\mathbf{k}]$ is the stiffness matrix of joining elements. Dependence of damping forces on the velocity of deformations $\{\dot{\mathbf{d}}(t)\}$ is described by the equation

$$
\left\{\mathbf{F}_{\mathbf{d}}(\mathrm{t})\right\}=[\mathbf{b}] \cdot\{\dot{\mathbf{d}}(t)\}
$$

where [b] is damping matrix. The derivation with respect of time $t$ is denoted by dot above the symbol. Resulting forces in jointing members in action on mass objects are

$$
\left\{\mathbf{F}_{\mathbf{J M}}(t)\right\}=-\left\{\mathbf{F}_{\mathbf{r e}}(t)\right\}-\left\{\mathbf{F}_{\mathbf{d}}(t)\right\}
$$

Sign (-) is due to the principle of action and reaction. From the forces in joining elements $\left\{\mathbf{F}_{\mathbf{J M}}(t)\right\}$ the static equivalents corresponding to individual degrees of freedom $\left\{\mathbf{F}_{\mathbf{S V}}(t)\right\}$ are calculated

$$
\left\{\mathbf{F}_{\mathbf{D F}}(t)\right\}=[\mathbf{A}] \cdot\left\{\mathbf{F}_{\mathbf{J M}}(t)\right\}
$$

The gravity forces $\left\{\mathbf{F}_{\mathbf{G}}\right\}$ and reactions in support $\left\{\mathbf{F}_{\mathbf{R S}}(t)\right\}$ must be added to the forces corresponding to individual degrees of freedom $\left\{\mathbf{F}_{\mathbf{D F}}(t)\right\}$. In this manner we obtain the complete vector of forces $\left\{\mathbf{F}_{\mathbf{R}}(t)\right\}$ acting on the computing model of vehicle

$$
\left\{\mathbf{F}_{\mathbf{R}}(t)\right\}=\left\{\mathbf{F}_{\mathbf{D F}}(t)\right\}+\left\{\mathbf{F}_{\mathbf{G}}\right\}+\left\{\mathbf{F}_{\mathbf{R S}}(t)\right\}
$$

The system of equations of motion describing the vibration of the computational model of vehicle is then expressed by the relation

$$
[\mathbf{m}] \cdot\{\ddot{\mathbf{r}}(t)\}=\left\{\mathbf{F}_{\mathbf{R}}(t)\right\}
$$

where $[\mathbf{m}]$ is mass matrix. After substituting for $\{\mathbf{r}(t)\}=\left[r_{1}(t), r_{2}(t), u(t)\right]^{\mathrm{T}}$,

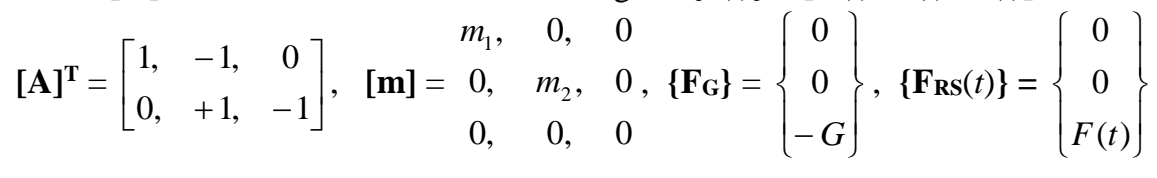


we obtain the system of ordinary differential equations describing the behaviour of the computational model and the equation for contact force

$$
\begin{gathered}
\ddot{r}_{1}(t)=\left\{-k_{1} \cdot\left[r_{1}(t)-r_{2}(t)\right]-b_{1} \cdot\left[\dot{r}_{1}(t)-\dot{r}_{2}(t)\right]\right\} / m_{1} \\
\ddot{r}_{2}(t)=\left\{+k_{1} \cdot\left[r_{1}(t)-r_{2}(t)\right]-k_{2} \cdot\left[r_{2}(t)-u(t)\right]+b_{1} \cdot\left[\dot{r}_{1}(t)-\dot{r}_{2}(t)\right]\right\} / m_{2} \\
F(t)=F_{s}(t)+F_{d}(t)=-G+k_{2} \cdot\left[r_{2}(t)-u(t)\right]
\end{gathered}
$$

The function $r_{1}(t)$ describes the time course of the vertical deflections of the upper mass $m_{1}$, the function $r_{2}(t)$ describes the time course of vertical deflections of the lower mass $m_{2}$ and the function $u(t)$ describes the time course of the road profile. The stiffness and damping constants are denoted as $k_{1}, k_{2}, b_{1}[4]$.

\section{Frequency response function}

Frequency response function $F R(p)$, where $p=\mathrm{i} . \omega$ is a complex number, is defined as a ratio of steady-state response and harmonic excitation. If the excitation is harmonic with uniting amplitude, then

$$
F R(p)=F R(\mathrm{i} \cdot \omega)=r_{\text {sts }}(t) /\left(h \cdot \mathrm{e}^{\mathrm{i} \cdot \omega \cdot t}\right)=r_{\text {sts }}(t) /\left(1 \cdot \mathrm{e}^{\mathrm{i} \cdot \omega \cdot t}\right)=r_{\text {sts }}(t) \cdot \mathrm{e}^{-\mathrm{i} \cdot \omega \cdot t}
$$

Frequency response function $F R(p)$ as the function of complex variable can be expressed as

$$
F R(p)=|F R(p)| \cdot \mathrm{e}^{\mathrm{i} \cdot \phi}
$$

where $|F R(p)|$ is the absolute value or the magnitude of the frequency response. We can express $r_{\mathrm{sts}}(t)$ from equation (10)

$$
F R(p)=r_{\text {sts }}(t) /\left(1 \cdot \mathrm{e}^{\mathrm{i} \cdot \omega \cdot t}\right) \quad \rightarrow \quad r_{\text {sts }}(t)=F R(p) \cdot \mathrm{e}^{\mathrm{i} \cdot \omega \cdot t}
$$

After substituting (11) to (12)

$$
r_{\text {sts }}(t)=|F R(p)| \cdot \mathrm{e}^{\mathrm{i} \cdot \phi} \cdot \mathrm{e}^{\mathrm{i} \cdot \omega \cdot t}=|F R(p)| \cdot \mathrm{e}^{\mathrm{i} \cdot(\omega \cdot t+\phi)}
$$

The graphic representation of frequency response is the frequency characteristic. The graphic representation of the absolute value (modulus) of frequency response is amplitude characteristic. The phase characteristic is the graphic representation of argument (phase) of frequency response in dependence on the frequency of harmonic excitation. The function $|F R(p)|^{2}$ is called power response factor (PRF).

The Laplace integral transform for the passing from time to frequency domain can be used. The Laplace picture of some function $r(t)$ will be denoted as $L\{r(t)\}=R(p)$. In this case $p=\mathrm{i} \cdot \omega$ is the complex number. The function $r(t)$ and their derivatives with the respect of time will be transformed as follows

$$
L\left\{r^{(n)}(t)\right\}=p^{n} \cdot R(p)-\sum_{i=1}^{n-1} p^{n-1-i} \cdot r^{(i)}(0+) \quad \text { for } n=1,2, \ldots \ldots
$$


The time functions $r_{1}(t), r_{2}(t)$ and $u(t)$ will be transformed on $R_{1}(p), R_{2}(p)$ and $U(p)$. The following designation can define 2 frequency responses

$$
\bar{r}_{i}=\frac{R_{i}(p)}{U(p)}, i=1,2
$$

By the Laplace transform of equations of motion and by defining the frequency responses we obtain the system of 2 equations in complex form for the function $\bar{r}_{i}(i=1,2)$. The matrix formulation is as follows

Generally

$$
[\mathrm{a}] \cdot\{\overline{\mathbf{r}}\}=\{\mathrm{RS}\}
$$

$$
a_{i k}=a_{i k, \mathrm{re}}+\mathrm{i} \cdot a_{i k, \mathrm{~m}}, \quad \bar{r}_{i}=\bar{r}_{i, \mathrm{re}}+\mathrm{i} \cdot \bar{r}_{i, \mathrm{~m}}, R S_{i}=R S_{i, \mathrm{re}}+\mathrm{i} \cdot R S_{i, \mathrm{im}}
$$

Similarly, the frequency response of a dynamic component of contact force is defined as

$$
\bar{F}_{d}=\frac{L\left\{F_{d}(t)\right\}}{U(p)}=k_{2} \cdot\left(\bar{r}_{2}-1\right)
$$

The Power Response Factors (PRF) of selected frequency functions at the speed of vehicle motion $10 \mathrm{~m} / \mathrm{s}$ are plotted on the following figures. The values of natural frequencies of the vehicle (calculated without damping) are introduced for better orientation: $f_{(1)}=1.06 \mathrm{~Hz}, f_{(2)}$ $=8.89 \mathrm{~Hz}$.

The frequency spectrum of the output signal can be obtained by multiplying the frequency response function of the system by the frequency spectrum of the input signal

$$
F S_{r}(p)=F R(p) \cdot F S_{u}(p)
$$

By introducing the power spectral densities of the input signal $P S D_{u}(\omega)$ and output signal $P S D_{r}(\omega)$ it can be written [4]

$$
\operatorname{PSD}_{r}(\omega)=|F R(p)|^{2} \cdot P S D_{u}(\omega) \text { or } \quad|F P(p)|^{2}=\operatorname{VSH}_{r}(\omega) / V S H_{h}(\omega)
$$

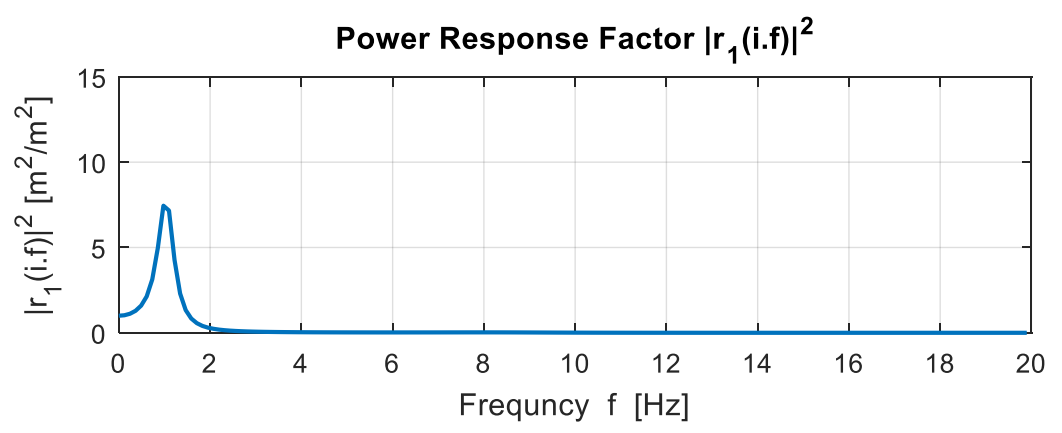



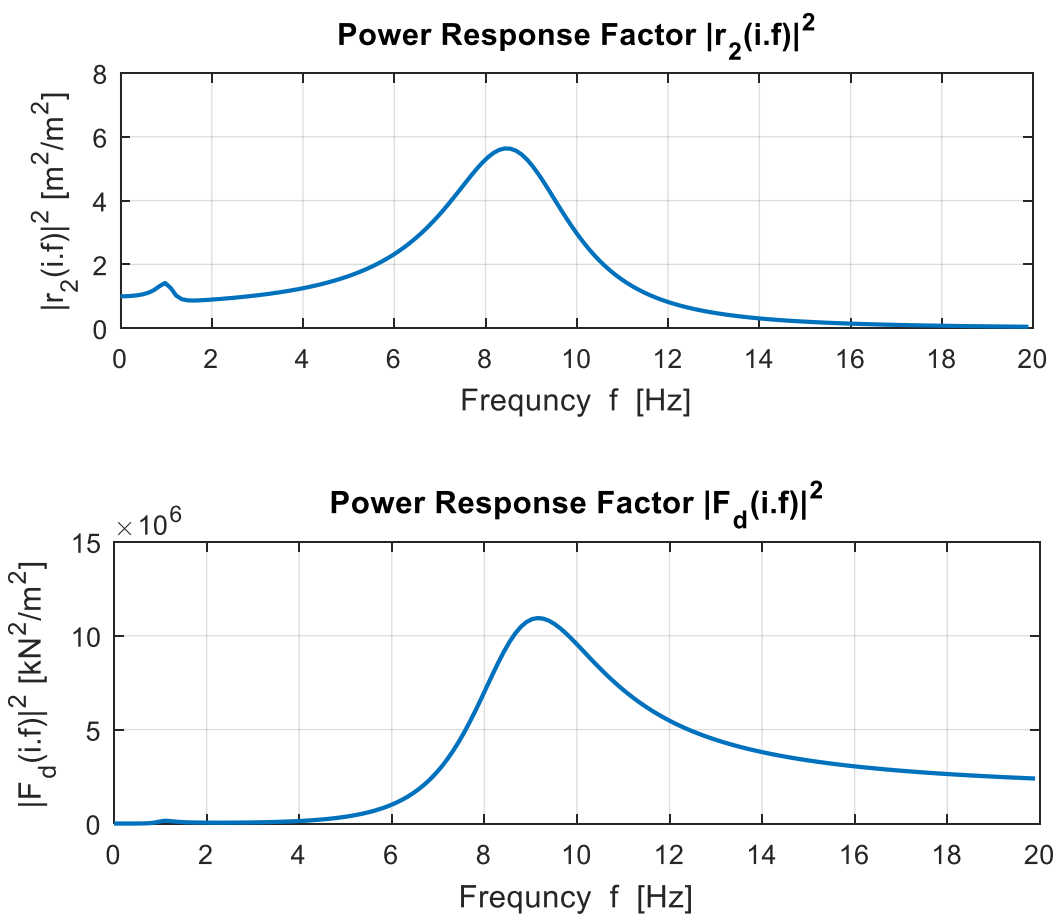

Fig. 2. Power response factor of selected frequency functions at the vehicle.

\section{Computational model of the road}

Assume the computational model of the road as rigid pavement with stochastic of road profile. Standard ISO 8608 [5] sorts the roads according to the quality of the surface into 8 categories marked A to $\mathrm{H}$. The values of the constant $S_{u}\left(\Omega_{0}\right)$ for the reference path angular frequency $\Omega_{0}=1.0[1 / \mathrm{m}]$ are given in Table 1 . For numerical simulations of vehicle movement along the road, it is necessary to generate a random longitudinal road profile based on the known power spectral density of unevenness $S_{u}(\Omega)$. There is a problem here. During measurement of random unevenness, only amplitude characteristics are evaluated and the phase characteristics are not evaluated. At the realization of reverse process one (phase) characteristic is missing. To generate a random road profile the following formula can be used

$$
u(x)=\sum_{i}^{N} \sqrt{2 S_{u}\left(\Omega_{i}\right) \cdot \Delta \Omega} \cdot \cos \left(\Omega_{i} \cdot x+\varphi_{i}\right)
$$

The angle $\varphi_{i}$ is the phase shift angle randomly divided into the interval $(0 ; 2 \pi)$. It is generated according to the uniform distribution.

The stochastic road profile generated by (21) for category B is shown in Fig. 3. It represents the source of kinematic excitation of vehicle. The Power Spectral Density of the road profile is shown in Fig. 4. 
Table 1. Classification of roads according to height unevenness [5].

\begin{tabular}{|c|c|c|c|}
\hline \multirow{3}{*}{$\begin{array}{c}\text { Class of } \\
\text { road }\end{array}$} & \multicolumn{3}{|c|}{ Degree of unevenness } \\
\cline { 2 - 4 } & \multicolumn{3}{|c|}{$S_{u}\left(\Omega_{0}\right)\left[\mathrm{m}^{2} /(\mathrm{rad} / \mathrm{m})\right]$ at $\Omega_{0}=1 \mathrm{rad} / \mathrm{m}$} \\
\hline A & - & $1 \cdot 10^{-6}$ & $2 \cdot 10^{-6}$ \\
\hline B & $2 \cdot 10^{-6}$ & $4 \cdot 10^{-6}$ & $8 \cdot 10^{-6}$ \\
\hline $\mathrm{C}$ & $8 \cdot 10^{-6}$ & $16 \cdot 10^{-6}$ & $32 \cdot 10^{-6}$ \\
\hline $\mathrm{D}$ & $32 \cdot 10^{-6}$ & $64 \cdot 10^{-6}$ & $128 \cdot 10^{-6}$ \\
\hline $\mathrm{E}$ & $128 \cdot 10^{-6}$ & $256 \cdot 10^{-6}$ & $512 \cdot 10^{-6}$ \\
\hline $\mathrm{F}$ & $512 \cdot 10^{-6}$ & $1024 \cdot 10^{-6}$ & $2048 \cdot 10^{-6}$ \\
\hline $\mathrm{G}$ & $2048 \cdot 10^{-6}$ & $4096 \cdot 10^{-6}$ & $8192 \cdot 10^{-6}$ \\
\hline $\mathrm{H}$ & $8192 \cdot 10^{-6}$ & $16384 \cdot 10^{-6}$ & - \\
\hline \multicolumn{3}{|c}{} \\
\hline
\end{tabular}

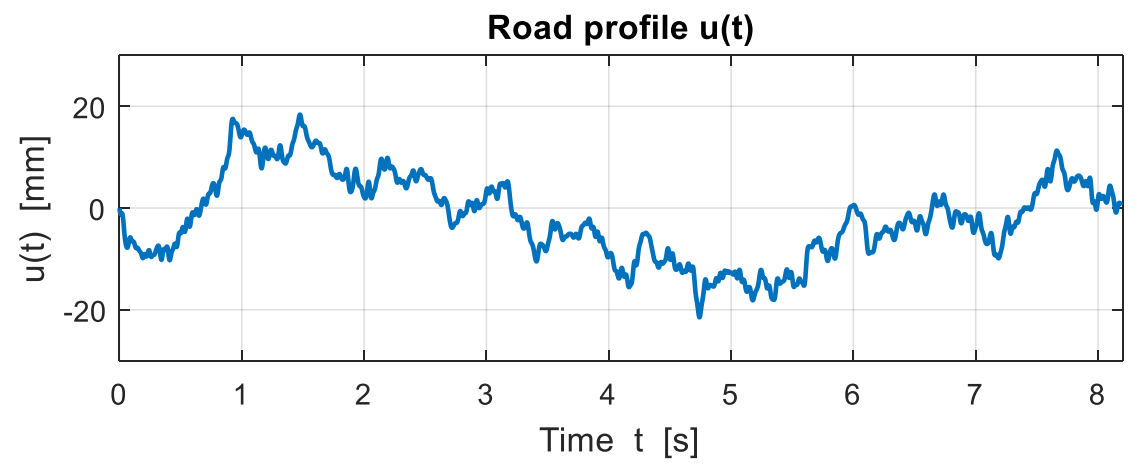

Fig. 3. The stochastic road profile.

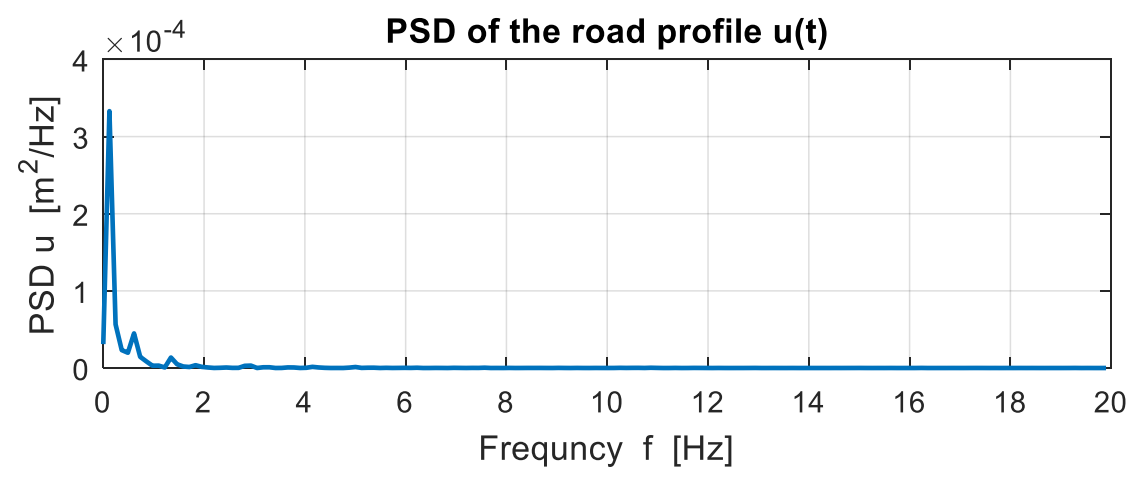

Fig. 4. Power Spectral Density. 


\section{Solution of the dynamic response in frequency domain}

Solution of dynamic response in frequency domain can be carried out in many ways. The simplest way is to obtain the Power Spectral Density of input signal, in our case the Power Spectral Density of road unevenness. Then to calculate the Frequency Response of analysed dynamical system specifically the Power Response Factors of individual quantities. And simply multiply the Power Response Factors of individual quantities by the Power Spectral Density of input signal, in our case by the Power Spectral Density of the road profile. The results thus obtained are shown in Fig. 5.
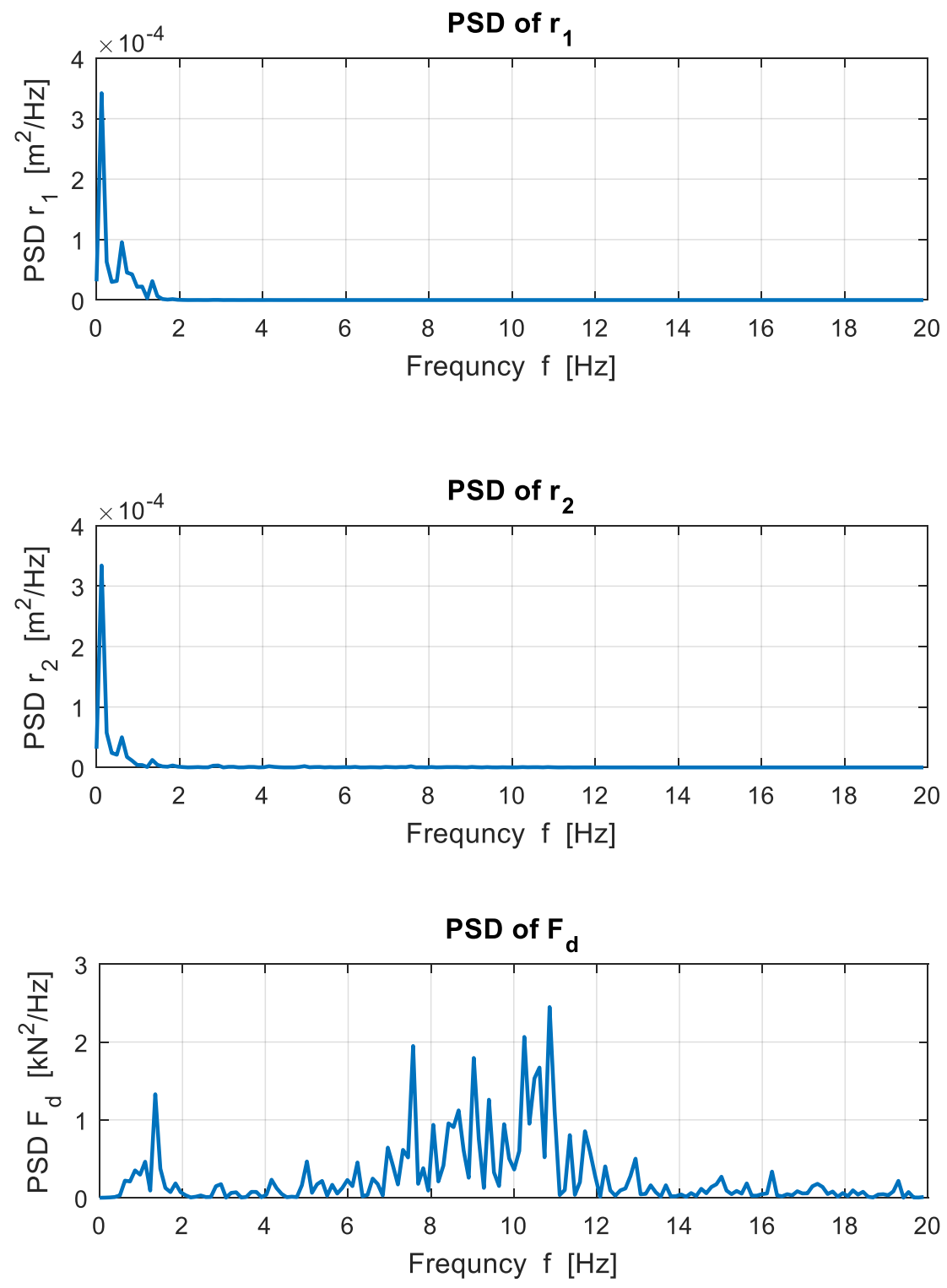

Fig. 5. Power Spectral Density of the road profile. 


\section{Conclusion}

Vehicle vibration induced by its motion along stochastic road profile can be solved in time or in the frequency domain. When we know the modules of FRF or the Power Response Factors (PRF) of vehicle model the calculation of vehicle response in the frequency domain is fast and efficient. In PSD of road profile predominate low frequencies (long waves). Upper and lower masses $m_{1}$ and $m_{2}$ sensitively respond to these low frequencies. The dynamic component of contact force $F_{d}$ sensitively responds to frequencies close to the second natural frequency of vehicle computational model, frequency interval approximately from 7 to 11 $\mathrm{Hz}$.

This paper was supported by the Grant National Agency VEGA of the Slovak Republic (grant No. $1 / 0006 / 20)$.

\section{References}

1. G. Verros, S. Natsiavas, G. Stepan, Control and dynamics of quarter car models with dual-rate damping, J Vib Control, 6 (2000)

2. M. Gobbi, G. Mastinu, Analytical description and optimization of the dynamic behaviour of passively suspended road vehicles, J Sound Vib, 245 (2001)

3. S. Turkay, H. Akcay, A study of random vibration characteristics of the quarter car model, J Sound Vib, 282 (2005)

4. J. Melcer, G. Lajčáková, I. Martinická, J. Králik, Dynamics of transport structures, (EDIS, Žilina, 2016, in Slovak)

5. ISO 8608, Mechanical vibration - road surface profiles - reporting of measured data. International standard, 1995 